\title{
A influência da espiritualidade na terapêutica e prognóstico dos pacientes com transtornos mentais
}

\author{
The influence of spirituality on therapy and prognosis of patients with mental disorders \\ La influencia de la espiritualidad en la terapia y el pronóstico de pacientes con trastornos \\ mentales
}

Lorena Novaes Gomes $^{1 *}$, Isabela Cornelio de Freitas Rodrigues ${ }^{2}$, Silvio Matheus Azevedo Rocha ${ }^{3}$, Wanessa Gonçalves Alves ${ }^{4}$, Letícia Lourenço Botelho ${ }^{5}$, Ana Clara Silva de Melo Franco ${ }^{2}$, Daiane Silvério Santana ${ }^{6}$, Stefany Fernandes Labuda ${ }^{2}$, Letícia Santana Ferreira Gonçalves ${ }^{7}$, Gustavo Fonseca Genelhu Soares ${ }^{8}$.

\section{RESUMO}

Objetivo: Analisar a relação da espiritualidade e o seu impacto no prognóstico e terapêutica dos três transtornos psiquiátricos mais prevalentes: depressão, ansiedade e transtorno afetivo bipolar. Revisão bibliográfica: A espiritualidade é um termo complexo, mas está relacionada à conexão entre o mundo físico e o espiritual. Estudos expostos no artigo estabelecem a relação da espiritualidade com o transtorno afetivo bipolar, como um fator positivo quando associada a psicofarmacoterapia, entretanto a espiritualidade depende da concepção individual e se internalizada pelo contexto negativo pode ser fonte de angústia e obstáculo ao tratamento. Ademais, a espiritualidade tem sido explorada como terapêutica no transtorno de ansiedade e depressão, os resultados são promissores e geram um aumento da qualidade de vida. Considerações finais: Diante do exposto, a espiritualidade exerce influência positiva ao tratamento e prognóstico de pacientes com transtornos mentais e é um fator protetivo importante. Assim, os profissionais de saúde devem estar capacitados para observar quando a espiritualidade está trazendo melhora ou nos casos que a própria interpretação da crença prejudica o manejo do paciente.

Palavras-chave: Espiritualidade, Depressão, Transtornos mentais.

\begin{abstract}
Objective: Analyze the relationship between spirituality and its impact on the prognosis and treatment of the three most prevalent psychiatric disorders: depression, anxiety and bipolar affective disorder. Literature review: Spirituality is a complex term but it is related to the connection between the physical and the spiritual world. Studies exposed in the article establish the relationship between spirituality and bipolar affective disorder, as a positive factor when associated with psychopharmacotherapy, however, spirituality depends on the individual conception and if internalized by the negative context, it can be a source of anguish and an obstacle to treatment. Furthermore, spirituality has been explored as a therapy for anxiety and depression disorders, the results are promising and generate an increase in quality of life. Final considerations: Given the above, spirituality has a positive influence on the treatment and prognosis of patients with mental disorders and is an important protective factor. Thus, health professionals must be able to observe when spirituality is bringing improvement or in cases where the interpretation of the belief affects the patient's management.
\end{abstract}

Key words: Spirituality, Depression, Mental disorders.

\footnotetext{
${ }^{1}$ Universidade Federal dos Vales do Jequitinhonha e Mucuri (UFVJM), Teófilo Otoni - MG.

*E-mail: lorena.novaes@ufvjm.edu.br

2 Pontifícia Universidade Católica de Minas Gerais (PUC MINAS), Betim - MG.

3 Universidade Tiradentes (UNIT), Aracajú - SE.

4 Universidade de Rio Verde (UNIRV), Aparecida de Goiânia - GO.

${ }^{5}$ Centro Universitário de Valença (UNIFAA), Valença - RJ.

${ }^{6}$ Universidade de Rio Verde (UNIRV), Formosa - GO.

${ }^{7}$ Centro Universitário do Planalto Central (UNICEPLAC), Brasília - DF.

${ }^{8}$ Centro Universitário de Caratinga (UNEC), Caratinga - MG.
} 


\section{RESUMEN}

Objetivo: Analizar la relación entre la espiritualidad y su impacto en el pronóstico y tratamiento de los tres trastornos psiquiátricos más prevalentes: depresión, ansiedad y trastorno afectivo bipolar. Revisión de la literatura: La espiritualidad es un término complejo pero está relacionado con la conexión entre el mundo físico y el espiritual. Los estudios expuestos en el artículo establecen la relación entre la espiritualidad y el trastorno afectivo bipolar, como factor positivo cuando se asocia a la psicofarmacoterapia, sin embargo, la espiritualidad depende de la concepción individual y si es interiorizada por el contexto negativo, puede ser fuente de angustia y obstáculo. al tratamiento. Además, la espiritualidad ha sido explorada como terapia para los trastornos de ansiedad y depresión, los resultados son prometedores y generan un aumento en la calidad de vida. Consideraciones finales: Dado lo anterior, la espiritualidad tiene una influencia positiva en el tratamiento y pronóstico de los pacientes con trastornos mentales y es un factor protector importante. Por lo tanto, los profesionales de la salud deben poder observar cuándo la espiritualidad está mejorando o en los casos en que la interpretación de la creencia afecta el manejo del paciente.

Palabras clave: Espiritualidad, Depresión, Trastornos mentales.

\section{INTRODUÇÃO}

A palavra espiritualidade vem do latim spiritus que significa elevação, transcendência e sublimidade (GOMES MV, et al., 2019). Assim, pode-se entender a espiritualidade como uma parte complexa e multidimensional, um estado de coragem que proporciona a conexão entre o mundo físico e o mundo espiritual. Essa sublimidade está correlacionada com experiências, sentimentos e emoções. Outrossim compreende-se que a manifestação está associada a princípios individuais e culturais, sejam eles fatores ambientais, religiosos e/ou artísticos (ABUCHAIM SCB, 2018).

A ciência e a espiritualidade foram separadas por cerca de 500 anos, pois presumiam que a ciência só poderia ser desenvolvida se não estivesse interconectada com a religião, uma vez que esta poderia influenciar, de forma autoritária, na formação de pensamentos e no livre arbítrio. Em contrapartida, atualmente, a produção de conhecimento atrelada à religiosidade tem se tornado cada vez mais frequentes por meio da pesquisa científica (KOENIG HG, et al., 2012). Tal fato é relevante visto que a fé, associada de forma positiva, pode auxiliar no tratamento, possibilitando que o paciente tenha uma melhor aceitação em relação à doença (DELLACQUA MN, et al., 2019).

Segundo a literatura, nota-se que grande parte das pessoas que possuem a espiritualidade desenvolvida obtiveram um progresso expressivo na terapêutica de transtornos mentais, dentre eles, ansiedade, depressão e transtorno bipolar. Por conseguinte, conclui-se a transcendência como uma extensão importante da prática clínica, uma vez que exterioriza maior compreensão e adaptação à enfermidade, gerando paz, autoconfiança e perdão, e uma imagem positiva de si mesmo (MONTEIRO DD, 2020).

A espiritualidade e a prática religiosa estão associadas e impactam a saúde mental através de comportamentos, estilo de vida e crenças sociais, ou seja, quanto maior o nível de envolvimento maior a satisfação e o bem-estar psicológico (SALIMENA AM, et al., 2016). Desse modo, foram analisados estudos, por profissionais da saúde, que demonstravam as maiores chances de reinserção social, apoio afetivo ou social, que pacientes adeptos a crenças apresentaram em relação aos que se consideravam céticos (REINALDO AMS e SANTOS RLF, 2016).

Somado a isso, notou-se que os pacientes com a espiritualidade desenvolvida apresentavam uma redução no uso de medicamentos e uma melhor qualidade de vida quando comparados àqueles os quais não possuíam nenhuma crença ou religião. Ademais pacientes com transtornos mentais relataram que a experiência com a sublimidade os proporcionou força e esperança para a vida quando associada à terapêutica e a rede de apoio, portanto, a espiritualidade é um aparato para prosseguir mesmo em meio às limitações da doença (REINALDO AMS e SANTOS RLF, 2016).

Diante do exposto, define-se como objetivo do presente trabalho evidenciar, por meio da revisão narrativa, a influência da espiritualidade na terapêutica e prognóstico de pacientes com transtornos mentais. 


\section{REVISÃO BIBLIOGRÁFICA}

\section{Transtorno afetivo bipolar}

O Transtorno Afetivo Bipolar (TAB) é responsável por impactar a vida dos pacientes com possíveis comprometimentos em relações sociais, familiares e atuação profissional. Sintomas depressivos no transtorno bipolar são os que mais podem afetar a funcionalidade e qualidade de vida do indivíduo. Recentemente, observou-se benefícios ao complementar a psicofarmacoterapia com diferentes abordagens psicossociais na busca de frear a progressão da doença, alcançar a recuperação funcional e sintomática e enfrentar flutuações de humor nos episódios de depressão e mania (STROPPA A, et al., 2018).

Neste contexto, a espiritualidade tem sido descrita como forma de auxiliar no tratamento psicoemocional dos portadores deste transtorno mental e contribuir para melhoria da saúde emocional e física. Embora já se reconheça a importância de considerar os aspectos espirituais e religiosos no manejo integral ao paciente, essas questões ainda são negligenciadas pelo foco ao tratamento tradicional medicamentoso e restrito à doença (SALIMENA AM, et al., 2016).

Em um estudo longitudinal analisaram 334 pacientes veteranos de guerra que sofriam de TAB em um Centro de Saúde Mental averiguando assim, o envolvimento enfático de práticas religiosas, meditação, oração individual e crenças espirituais, sendo que, entre os indivíduos que estavam na fase mista de TAB foram evidenciados um maior nível de oração, em comparação com os pacientes na fase de estabilização clínica. Assim, nesse estudo é importante destacar que pacientes que vivenciavam sua própria fé de maneira íntima relataram que isso poderia ter um impacto positivo no curso de seu transtorno. Nesse contexto, a crescente demonstração em relação terapêutica da meditação e da espiritualidade na psiquiatria deve ser considerada como um enfoque importante do tratamento (FAZIO P, et al., 2015).

Huguelet $P$, et al. (2016), realizaram um estudo para explorar o significado espiritual da vida entre 175 pacientes com diversos transtornos mentais na França e Suíça. Neste estudo, 25\% dos pacientes com transtorno mental grave atribuíram um significado essencial da religião e espiritualidade em suas vidas. Porém, a prevalência da espiritualidade variava de acordo com o diagnóstico. Apesar da importância que a espiritualidade pode ter $\mathrm{TAB}$, apenas $9 \%$ dos pacientes com TAB relataram que a espiritualidade e religião teriam papéis significativos em suas vidas. De fato, como estudos qualitativos demonstraram, pacientes com TAB relataram dificuldades para interpretar suas experiências espirituais em estados maníacos e depressivos.

Em um estudo transversal publicado em 2017, em um total de 369 pacientes foram incluídos (Áustria: $\mathrm{n}=$ 189, Japão: $n=180$ ), consistindo de 112 pacientes com esquizofrenia paranoide, 120 pacientes com transtorno bipolar I e 137 controles saudáveis, os atributos clínicos foram similares entre os dois países, exceto em relação à duração da doença e aos sintomas depressivos. Os indivíduos japoneses com esquizofrenia tiveram uma duração mais longa da doença em comparação com pacientes bipolares austríacos. A distribuição das denominações revela que uma proporção maior de indivíduos austríacos relatou pertencer a uma denominação cristã, enquanto um número maior de pacientes japoneses indicou ser budista. No entanto, não foi constatado uma maior frequência e importância de atividades religiosas / espirituais de alcances significantes em pacientes com esquizofrenia paranoide ou TAB (MIZUNO Y, et al., 2017).

Em 2018, publicou-se estudo prospectivo de dois anos para analisar o efeito do envolvimento religioso no curso do TAB e enfrentamento dos sintomas de depressão, mania e repercussões na qualidade de vida dos pacientes. Neste estudo realizado com 168 pessoas, o coping religioso, ou seja, as maneiras as quais os pacientes utilizam crenças e comportamentos religiosos para se adaptar ao estresse e situações desafiadoras, influenciaram as mudanças na qualidade de vida ao longo do tempo. Por um lado, a utilização de estratégias positivas como buscar em Deus lições ao ser confrontado com problemas ou fazer o possível e deixar o que não se tem controle para a responsabilidade divina previu um aumento na qualidade de vida. Por outro lado, quando o indivíduo assumiu uma postura passiva, delegando a Deus a resolução de seus problemas ou atribuindo-os à punição divina, teve como resultado o declínio na qualidade de vida (STROPPA A, et al., 2018).

Desse modo, a espiritualidade pode ter importantes aspectos positivos com impacto na qualidade de vida, reforço de valores individuais e uma perspectiva de vida com maior significado, auxiliando no cuidado voltado 
à recuperação. No entanto, a espiritualidade depende da concepção individual e se internalizada pelo paciente em um contexto negativo, pode ser fonte de angústia e obstáculo ao tratamento (HUGUELET P, et al., 2016).

\section{Transtorno de ansiedade}

Inúmeros estudos têm sido desenvolvidos com o intuito de estabelecer uma relação concreta entre os transtornos de ansiedade e a religiosidade. Embora a maior parcela de pesquisas realizadas indique a existência de uma relação positiva entre os parâmetros estudados, alguns estudiosos apontam também a existência de efeitos contrários, nos quais a religiosidade atua de forma a criar transtornos ou de agravar aqueles já existentes (MONTEIRO DD, et al., 2020).

A corrente de pesquisa com maior proporção estatística indica a existência de uma relação positiva entre práticas religiosas e ansiedade. Ao abordar-se a saúde mental como um todo, estudos indicam que indivíduos adeptos a uma corrente religiosa e praticante de rituais religiosos possuem menores taxas de depressão, ansiedade, tentativas de suicídio e abuso de substância, e, ao mesmo tempo, possuem melhor qualidade de vida. Dados de uma pesquisa realizada com 129 participantes do Mato Grosso do Sul, em situação de vulnerabilidade social, indicaram que aqueles declaradamente religiosos apresentavam menos ansiedade. Além disso, foi questionado aos participantes sobre sua opinião a respeito do papel da religião no cuidado em saúde mental. A maior parte dos indivíduos (87.9\%) afirmou achar que a religiosidade possui influência sobre a saúde física e mental e $84,4 \%$ acreditavam que a ausência de fé e religião influenciavam no aparecimento de sintomas depressivos e de ansiedade (GONÇALVES LM, et al., 2018).

Apesar de a maioria das pesquisas abordarem a religião católica, alguns trabalhos buscam ampliar essa análise, abordando diferentes manifestações de religiosidade. Uma pesquisa realizada no Japão em um grupo de psicoterapia baseado na filosofia budista, denominado "Happy Smile Clinic", por exemplo, indicou resultados semelhantes aos observados para indivíduos católicos, comprovando uma redução de sintomas depressivos e de ansiedade em pacientes religiosos e praticantes da filosofia budista. Além disso, foi realizado um acompanhamento desses indivíduos pelo período de três meses. Ao final deste prazo, foi constatada a continuidade dos resultados observados no início do processo (CHIDA Y, et al., 2016).

De forma geral, comprova-se que indivíduos mais religiosos apresentam menos ansiedade e melhor qualidade de vida, além de melhores desfechos psiquiátricos. Considerando o sentido da religião na vida dos indivíduos, compreende-se que a concepção sobre o desenvolvimento de uma "parceria" com Deus atua fortemente no sentido de reduzir transtornos de humor, como o transtorno de ansiedade generalizada e a depressão. Esse efeito positivo da religião pode ser, também, explicado pelo desenvolvimento de sentimentos positivos, como altruísmo, gratidão, perdão, além de atender à necessidade da existência de um sentido para a vida. Um outro fator de relevância estudado é o efeito da mudança de religião nos transtornos de ansiedade. Estudos indicam que o ato de alterar a filosofia religiosa de escolha acarreta efeitos negativos, levando à redução da autoestima e da qualidade de vida, além de aumentar a ansiedade. Com isso, pode-se compreender o quão complexa a relação religiosidade-ansiedade pode ser, podendo a religião ser tanto parte da solução, quanto do problema (MOREIRA-ALMEIDA A, et al., 2014).

Por outro lado, apesar da existência de efeitos positivos da religiosidade no enfrentamento da ansiedade, não se pode omitir a possibilidade de esta agravar este transtorno. A crença inabalável em alguns dogmas religiosos e a não maleabilidade de certas práticas por parte dos adeptos, pode levar ao aumento da ansiedade pela indução de medo e culpa. Um exemplo disso pode ser observado entre jovens homossexuais religiosos. A concepção religiosa de que a homossexualidade é um pecado, leva muitos jovens a culparemse por não atenderem às expectativas impostas pela religião, aumentando o sentimento de medo com relação às possíveis consequências de seus atos. Com isso, pode ocorrer o aumento da ansiedade, assim como de outros transtornos. Dentre os possíveis aspectos negativos da religiosidade no que tange à ansiedade, podese citar o desenvolvimento de rigidez e dependência excessiva de leis e regras, além de enfatizar culpa e pecados, reduzindo a autonomia. Somado a isso, a concepção de que Deus pode ser a resposta para todos os problemas, pode levar os indivíduos a atrasarem seu processo de busca por ajuda profissional, o que pode levar à piora do quadro psiquiátrico (STEWART C, et al., 2019). 
Dessa forma, pode-se concluir que a religiosidade e a existência de fé entre indivíduos portadores de transtornos de ansiedade contribuem para mitigar efeitos da doença, independentemente da corrente religiosa seguida. No entanto, não se pode ignorar possíveis efeitos negativos da prática religiosa. Essa contradição pode ser explicada pela reduzida produção científica existente, além do baixo número de indivíduos acompanhados (STEWART C, et al., 2019).

\section{Depressão}

A depressão tem como medidas terapêuticas a psicoterapia cognitivo-comportamental e o uso de medicamentos antidepressivos. A primeira tem mostrado resultados positivos entre os pacientes depressivos (LI JM, et al.,2018). Já a terapia medicamentosa, embora apresente resposta satisfatória na redução dos sintomas, não é efetiva ou provoca efeitos colaterais em cerca de $50 \%$ dos pacientes (TRIVEDI MH, et al., 2006). Nesse sentido, há alguns anos, a espiritualidade e a religiosidade têm sido exploradas na terapêutica e no prognóstico dos pacientes com depressão. A R/E tem sido associada a um aumento na qualidade de vida geral (CARNEIRO EM, et al., 2020).

A espiritualidade tem mostrado um importante fator de redução da sintomatologia, do risco de suicídio e do sofrimento psicológico em pacientes com depressão. Além disso, tem sido associada a uma maior adesão ao tratamento (FERREIRA LF, et al., 2020). A ideação suicida e a depressão têm uma menor prevalência em pessoas que possuam algum nível de espiritualidade ou religiosidade (HAFIZI S, et al., 2014). Sendo assim, a espiritualidade é tida como predita de uma menor gravidade da depressão e, consequentemente, uma melhor qualidade de vida (BONELLI R, et al., 2012; KIM N, et al., 2015).

Em estudo realizado em 2015, a relação religiosidade / espiritualidade foi avaliada em pacientes deprimidos internados. Cerca de 143 pacientes internados em hospital psiquiátrico foram separados em grupos de alta religiosidade e baixa religiosidade. Informações sobre medidas clínicas, religiosidade, resiliência e qualidade de vida foram coletadas. Pacientes com religiosidade, na alta, apresentaram uma maior qualidade de vida, uma maior resiliência e uma menor chance de suicídio (MOSQUEIRO B, et al., 2015).

Em outro estudo, também realizado em 2015, 232 pacientes ambulatoriais com transtornos depressivos foram colocados à avaliação sobre sintomas psicológicos, religiosidade e espiritualidade. Foi utilizada uma escala clínica global de melhoria da impressão (CGI-1) para avaliação durante 6 meses. Ao final do estudo, foi visto que a espiritualidade é um fator preditor significativo para respostas favoráveis ao tratamento de pacientes com depressão (KIM N, et al., 2015).

Em 2012, um estudo prospectivo de 10 anos com 114 participantes sendo 72 adultos filhos de pais deprimidos e 42 filhos de não deprimidos avaliou o poder da religiosidade / espiritualidade em diminuir o risco de depressão. Foram coletadas informações sobre importância pessoal da religião ou espiritualidade, frequência de participação em serviços religiosos e denominação. Ao final do estudo, foi visto que os participantes que classificaram a religião ou a espiritualidade como altamente importantes tinham uma chance de estarem deprimidos $73 \%$ menor do que o grupo que classificou a religião ou a espiritualidade como algo não tão importante (MILLER L, et al., 2011).

Em 2018, pesquisadores estudaram os efeitos da religiosidade em pacientes idosos com depressão e insuficiência cardíaca, cerca de 93 pacientes separados em grupos experimental e controle foram avaliados através de checklists, questionários de satisfação com a vida do LSI-Z e o questionário de depressão de Beck. O grupo experimental foi conduzido em um programa religioso de 6 sessões e, após o período de testes, viuse que o índice de satisfação com a vida foi maior no grupo de teste. Dessa forma, os pesquisadores concluíram que a intervenção espiritual/religiosa foi útil na diminuição da depressão (ABDI A, et al., 2018).

Uma revisão sistemática da literatura realizada em 2019, analisou pesquisas sobre a religiosidade e a espiritualidade associadas com medidas neurobiológicas, por meio de EEG, neuroimagem funcional e neuroimagem estrutural. Além disso, esses estudos avaliaram uma parcela significativa das religiões, comportamentos e realizou diversas metodologias. De forma geral, a revisão demonstrou que, regiões cerebrais de ordem superior, ligadas às emoções e aos sentimentos, acessam uma reserva neural que origina o processo de neuroplasticidade. Dessa forma, uma reserva neural maior poderia apoiar uma reserva 
cognitiva aprimorada que permite que as pessoas com maior religiosidade / espiritualidade sejam mais resilientes, tenham redução de pensamentos autorreferencial e influência significativamente na patogênese e na terapêutica da depressão (RIM Jl, et al., 2019).

Nessa perspectiva, conclui-se que de forma geral os estudos convergem para a religiosidade e a espiritualidade como fatores capazes de interferirem nos processos de adoecimento e de cura (ABDALEATI NS, et al., 2016). Dessa forma, abordar a dimensão espiritual e religiosa permite a redução significativa dos sintomas depressivos e auxílio na terapêutica da depressão.

\section{CONSIDERAÇÕES FINAIS}

Com base nas informações analisadas na literatura aferiu-se que a influência da espiritualidade na terapêutica e no prognóstico de pacientes com transtornos mentais tem valor positivo quando praticada de forma maleável, na busca de conforto e sentido. O bem-estar psicológico, apoio afetivo e sentimentos positivos genuínos que trazem prazer para a vida beneficia os pacientes na melhora da qualidade de vida e até possível redução no uso de medicamentos, desde que acompanhados por um profissional adequado. Sendo assim, o paciente não deve substituir o tratamento designado pelas crenças espirituais ou enxergá-las como inflexíveis, o que pode por sua vez prejudicar o estado de saúde mental. Nesse sentido, profissionais do âmbito de saúde mental devem ser capacitados para entender quando a espiritualidade está trazendo melhora no tratamento e manejar os casos em que a interpretação da crença prejudica o prognóstico.

\section{REFERÊNCIAS}

1. ABDALEATI NS, et al. Religiousness and mental health: systematic review study. Journal of Religion and Health, 2016; 55: 1929-1937.

2. ABDI A, et al. The Effect of Religion Intervention on Life Satisfaction and Depression in Elderly with Heart Failure. J Relig Health, 2019; 58: 823-832.

3. ABUCHAIM SCB. Espiritualidade/religiosidade como recurso terapêutico na prática clínica: concepção dos estudantes de graduação em medicina da escola paulista de medicina - Universidade Federal de São Paulo. Ensino em Ciência da Saúde. 2018; 74.

4. BONELLI R, et al. Religious and Spiritual Factors in Depression: Review and Integration of the Research. Depression Research and Treatment, 2012: 1-8.

5. CARNEIRO EM, et al. Religiosidade/Espiritualidade, Indicadores de Saúde Mental e parâmetros hematológicos de profissionais de enfermagem. Revista de Enfermagem e Atenção à Saúde, 2020; 9(1).

6. CHIDA Y, et al. A Novel Religious/Spiritual Group Psychotherapy Reduces Depressive Symptoms in a Randomized Clinical Trial. J Relig Health, 2016; 55, 1495-1506.

7. DELLACQUA MN, et al. Panorama sobre espiritualidade e saúde: o que a literatura científica aponta sobre o tema nos últimos 5 anos? Res., Soc. Dv., 2019; 8(7): e10871103.

8. FAZIO P, et al. Religiosidade e espiritualidade em pacientes com transtorno bipolar. Jornal Internacional de Psiquiatria na Prática Clínica, 2015; 19(4): 233-7.

9. FERREIRA LF, et al. A Influência da Espiritualidade e da Religiosidade na Aceitação da Doença e no Tratamento de Pacientes Oncológicos: Revisão Integrativa da Literatura. Rev. Bras. Cancerologia, 2020; 66(2).

10. GOMES MV, et al. "À espera de um milagre": espiritualidade/religiosidade no enfrentamento da doença falciforme. Revista Brasileira de Enfermagem, 2019; 72(6): 1632-9.

11. GONÇALVES LM, et al. Spirituality, Religiosity, Quality of Life and Mental Health Among Pantaneiros: A Study Involving a Vulnerable Population in Pantanal Wetlands, Brazil. J Relig Health, 2018; 57: 2431-2443.

12. HAFIZI S, et al. Borderline Personality Disorder and Religion: A perspective from a Muslim country. Iranian Journal of Psychiatry, 2014; 9(3): 137-141.

13. HUGUELET $P$, et al. Spiritual Meaning in Life and Values in Patients With Severe Mental Disorders. The Journal of Nervous and Mental Disease, 2016; 204(6): 409-414.

14. KIM NY, et al. Effects of religiosity and spirituality on the treatment response in patients with depressive disorders. Compr Psychiatry, 2015; 60: 26-34.

15. KOENIG HG, et al. A history of religion medicine and healthcare. Handbook of Religion and Health. Nova York, Oxford University Press, 2012; 2.

16. LI JM, et al. Cognitive behavioral therapy for treatment-resistant depression: A systematic review and meta-analysis. Psychiatry Research, 2018; 268: 243-250.

17. MILLER L, et al. Religiosity and major depression in adults at high risk: a ten-year prospective study. Am J Psychiatry. $2012 ; 169(1): 89-94$.

18. MIZUNO Y, et al. Religiosidade e resiliência psicológica em pacientes com esquizofrenia e transtorno bipolar: um estudo transversal internacional. Acta Psychiatr Scand. SL. 2017. 
19. MONTEIRO DD, et al. Espiritualidade/Religiosidade e saúde mental no Brasil: Uma revisão. Boletim Academia Paulista de Psicologia, 2020; 40(98): 129-139.

20. MOREIRA-ALMEIDA A, et al. Clinical Implications of Spirituality to Mental Health: Review of Evidence and Practical Guidelines. Revista Brasileira de Psiquiatria, 2014; 36: 176-182.

21. MOSQUEIRO BP, et al. Intrinsic religiosity, resilience, quality of life, and suicide risk in depressed inpatients. Journal of affective disorders, 2015; 179: 128-133.

22. REINALDO AMS, SANTOS RLF. Religião e transtornos mentais na perspectiva de profissionais de saúde, pacientes psiquiátricos e seus familiares. Revista Saúde Debate, 2016; 40(110): 162-171.

23. RIM JI, et al. Current Understanding of Religion, Spirituality, and Their Neurobiological Correlates. Harv Rev Psychiatry, 2019; 27(5): 303-316.

24. SALIMENA AM, et al. Compreensão da espiritualidade para os portadores de transtorno mental: contribuições para o cuidado de enfermagem. Revista Gaúcha de Enfermagem, 2016; 37(3): 1-7.

25. STEWART C, et al. Review of the Effect of Religion on Anxiety. Int J Depress Anxiety, 2019; 2: 016.

26. STROPPA A, et al. Religiosity, depression, and quality of life in bipolar disorder: a two-year prospective study. Brazilian Journal of Psychiatry, 2018; 40(3): 238-243.

27. TRIVEDI MH, et al. Evaluation of outcomes with citalopram for depression using measurement-based care in $S T A R^{\star} D$ : implications for clinical practice. Am J Psychiatry, 2006; 163(1): 28-40. 